\title{
REILLY INEQUALITIES OF ELLIPTIC OPERATORS ON CLOSED SUBMANIFOLDS
}

\author{
RUSHAN WANG
}

\author{
(Received 5 November 2008)
}

\begin{abstract}
Using generalized position vector fields we obtain new upper bound estimates of the first nonzero eigenvalue of a kind of elliptic operator on closed submanifolds isometrically immersed in Riemannian manifolds of bounded sectional curvature. Applying these Reilly inequalities we improve a series of recent upper bound estimates of the first nonzero eigenvalue of the $L_{r}$ operator on closed hypersurfaces in space forms.
\end{abstract}

2000 Mathematics subject classification: primary 53C42, 53A10.

Keywords and phrases: closed submanifolds, elliptic operator, $r$-mean curvature.

\section{Introduction}

Let $M^{n}$ be a closed, connected and orientable $n$-dimensional Riemannian manifold isometrically immersed in the $m$-dimensional Euclidean space $\mathbb{E}^{m}(m>n)$, let $H$ denote the mean curvature of the immersion of $M^{n}$ into $\mathbb{E}^{m}$ and let $\lambda_{1}^{\Delta}$ denote the first nonzero eigenvalue of the Laplacian on $M^{n}$. In 1977, Reilly [6] proved

$$
\lambda_{1}^{\Delta} \leq \frac{n}{\operatorname{vol}(M)} \int_{M} H^{2} d M
$$

and a generalized Reilly inequality

$$
\lambda_{1}^{\Delta}\left(\int_{M} H_{r} d M\right)^{2} \leq n \operatorname{vol}(M) \int_{M} H_{r+1}^{2} d M, \quad 0 \leq r \leq n-1,
$$

where $H_{r}$ denotes the $r$-mean curvature of $M^{n}$. In 2004, Alias and Malacarne [2] considered an $L_{r}$ operator(see $\S 4$ ) on a closed, connected and orientable $n$-dimensional Riemannian manifold isometrically immersed in the $(n+1)$-dimensional Euclidean space $\mathbb{E}^{n+1}$; if $L_{r}$ is elliptic on $M^{n}$ for some $0 \leq r \leq n-1$, they proved a Reilly

The author was partially supported by grant number 60804044 of the NSFC.

(C) 2009 Australian Mathematical Publishing Association Inc. 0004-9727/2009 \$16.00 
inequality of $\lambda_{1}^{L_{r}}$ with $H_{r}, H_{s}$ as follows

$$
\lambda_{1}^{L_{r}}\left(\int_{M} H_{s} d M\right)^{2} \leq c_{r} \int_{M} H_{r} d M \int_{M} H_{s+1}^{2} d M, \quad 0 \leq s \leq n-1
$$

where $c_{r}=(n-r)\left(\begin{array}{l}n \\ r\end{array}\right)$.

When the ambient space is the Euclidean sphere $\mathbb{S}^{n+1}(1)$, let $X$ be the position vector of $M^{n}\left(\subset \mathbb{S}^{n+1}(1)\right)$ in $\mathbb{E}^{n+2}$, and $\langle$,$\rangle be the Euclidean metric on \mathbb{E}^{n+2}$. Alias and Malacarne [2] obtained the Reilly inequality including $\langle X, \eta\rangle$

$$
\lambda_{1}^{L_{r}}\left(\int_{M} H_{s}\langle X, \eta\rangle d M\right)^{2} \leq c_{r} \int_{M} H_{r} d M \int_{M} H_{s+1}^{2} d M, \quad 0 \leq s \leq n-1
$$

where $\eta$ is the gravity center vector of $M^{n}\left(\subset \mathbb{S}^{n+1}(1)\right)$ in $\mathbb{E}^{n+2}$.

There is no similar result for the case of hyperbolic space $\mathbb{H}^{n+1}(-1)$. Naturally we hope to obtain such unified inequalities only with $H_{r}, H_{S}$ for any simply connected space form $\mathbb{R}^{n+1}(c)$ of constant sectional curvature $c$.

On the other hand, when the ambient space is a Riemannian manifold $\left(\bar{M}^{m}, \bar{g}\right)(m>n)$ of sectional curvature bounded above by $c$, we define a tensor set on $M^{n}$ :

$$
\begin{gathered}
\mathcal{A}=\{T \mid T \text { is a symmetric positive-definite (1.1)-tensor } \\
\text { on } \left.M^{n} \text { such that } \operatorname{div}_{M} T=0\right\} .
\end{gathered}
$$

(Since $I_{n} \in \mathcal{A}$ we know that $\mathcal{A} \neq \emptyset$.) Given any $T \in \mathcal{A}$, Grosjean [5] considered the extrinsic upper bounds for the first nonzero eigenvalue of the elliptic operators $L_{T}$ defined on $\left(M^{n}, g\right)$ (that is, in terms of the second fundamental form of an isometric immersion of $\left(M^{n}, g\right)$ into $\left.\left(\bar{M}^{m}, \bar{g}\right)\right)$ of the form

$$
L_{T} u=\operatorname{div}_{M}\left(T \nabla^{M} u\right)
$$

where $u \in C^{\infty}(M), \operatorname{div}_{M}$ and $\nabla^{M}$ denote, respectively, the divergence and the gradient of the metric $g$ on $M^{n}$. Let $\phi$ be an isometric immersion of $\left(M^{n}, g\right)$ into $\left(\bar{M}^{m}, \bar{g}\right)$, and $\lambda_{1}^{T}$ be the first nonzero eigenvalue of the operator $L_{T}$, if $c \leq 0$ we assume that $\left(\bar{M}^{m}, \bar{g}\right)$ is simply connected, and if $c>0$ we assume that $\phi\left(M^{n}\right)$ is contained in a convex ball of radius less than or equal to $\pi / 4 \sqrt{c}$. Then he obtained

$$
\lambda_{1}^{T} \leq \frac{\sup _{M}\left|H_{T}\right|^{2}+\sup _{M} c(\operatorname{tr} T)^{2}}{\inf _{M} \operatorname{tr}(T)}
$$

and

$$
\left.\lambda_{1}^{T} \leq \sup _{M}\left(\left|H_{T}\right||H|+c(\operatorname{tr} T)\right), \quad \text { (if }\left|H_{T}\right|=\text { constant }\right)
$$

where $H_{T}(x)=\sum_{1 \leq i \leq n} h\left(T e_{i}, e_{i}\right),\left\{e_{i}\right\}_{1 \leq i \leq n}$ is an orthonormal basis of the tangent space $T_{x}(M)$ and $h$ is the second fundamental form of $\phi$. 
Inspired by the work of Alias and Malacarne [2] and Grosjean [5], we study these $T-S$ type upper bound estimates of the first nonzero eigenvalue of the $L_{T}$ operator, and prove the following results.

THEOREM 1.1. Let $\phi$ be an isometric immersion of a closed, connected Riemannian manifold $\left(M^{n}, g\right)(n \geq 2)$ into a complete Riemannian manifold $\left(\bar{M}^{m}, \bar{g}\right)(m>n)$ of sectional curvature bounded above by $c(c>0)$, we assume that $\phi\left(M^{n}\right)$ is contained in a convex ball of radius less than or equal to $\pi / 4 \sqrt{c}$, then we have

$$
\lambda_{1}^{T} \leq \frac{\int_{M} \operatorname{tr} T d v_{g}}{V}\left[c+\frac{1}{V} \frac{1}{\inf _{M}(\operatorname{tr} S)^{2}} \int_{M}\left|H_{S}\right|^{2} d v_{g}\right], \quad \text { for all } S \in \mathcal{A}
$$

where $V$ is the volume of $\phi\left(M^{n}\right)$. If equality holds, then $\phi(M)$ is contained in a geodesic hypersphere of $\bar{M}^{m}$. If $\left(\bar{M}^{m}, \bar{g}\right)$ is a constant curvature space of sectional curvature $c$ and $\phi(M)$ is contained in a geodesic hypersphere of $\bar{M}^{m}$, then equality holds.

THEOREM 1.2. Let $\phi$ be an isometric immersion of a closed, connected Riemannian manifold $\left(M^{n}, g\right)(n \geq 2)$ into a complete Riemannian manifold $\left(\bar{M}^{m}, \bar{g}\right)(m>n)$ of sectional curvature bounded above by $c$, if $c \leq 0$ we assume that $\left(\bar{M}^{m}, \bar{g}\right)$ is simply connected, and if $c>0$ we assume that $\phi(M)$ is contained in a convex ball of radius less than or equal to $\pi / 4 \sqrt{c}$. Then

$$
\lambda_{1}^{T} \leq \sup _{M}\left[c \operatorname{tr} T+\sup _{M}\left(\frac{\left|H_{T}\right|}{\operatorname{tr} S}\right)\left|H_{S}\right|\right] \quad \text { for all } S \in \mathcal{A} .
$$

If equality holds, then $\phi(M)$ is contained in a geodesic hypersphere of $\bar{M}^{m}$. If $\left(\bar{M}^{m}, \bar{g}\right)$ is a constant curvature space of sectional curvature $c$ and $\phi(M)$ is contained in a geodesic hypersphere of $\bar{M}^{m}$, then equality holds.

REMARK 1.3. Theorems 1.1 and 1.2 generalize Grosjean's [5] work. In fact, letting $S=T$ and $I$ respectively in (1.6), we obtain (1.3) and (1.4) easily.

Applying Theorems 1.1 and 1.2 to the $L_{r}$ operator, we derive the $H_{r}, H_{S}$ type upper bound estimates of its first nonzero eigenvalue of hypersurfaces isometrically immersed in space forms, which extend the corresponding results in $[1,2,8]$.

\section{Preliminaries}

Let $\phi$ be an isometric immersion of a compact, connected Riemannian manifold $\left(M^{n}, g\right)(n \geq 2)$ into a Riemannian manifold $\left(\bar{M}^{m}, \bar{g}\right)(m>n)$ of sectional curvature bounded above by $c$. If $c \leq 0$ we assume that $\left(\bar{M}^{m}, \bar{g}\right)$ is simply connected and if $c>0$ we assume that $\phi(M)$ is contained in a convex ball of radius less than or equal to $\pi / 4 \sqrt{c}$, and denote by $\nabla^{M}, \bar{\nabla}$ the gradients taken in $\left(M^{n}, g\right),\left(\bar{M}^{m}, \bar{g}\right)$, respectively. Using the fact that $\operatorname{div}_{M} T=0$, we know that $L_{T}$ is a self-adjoint and elliptic secondorder differential operator on $M^{n}$ with an equivalent form $L_{T} u=\operatorname{trace}(T \operatorname{Hess} u)$, 
it has discrete eigenvalues $0=\lambda_{0}<\lambda_{1} \leq \cdots$ where

$$
\lambda_{1}^{L_{T}}=\inf \left\{\frac{-\int_{M} f L_{T}(f) d v_{g}}{\int_{M} f^{2} d v_{g}}, f \in C^{\infty}(M), \int_{M} f d v_{g}=0\right\}
$$

is the first nonzero eigenvalue, and

$$
\begin{gathered}
\lambda_{i}^{L_{T}}=\inf \left\{\frac{-\int_{M} f L_{T}(f) d v_{g}}{\int_{M} f^{2} d v_{g}}, f \in C^{\infty}(M) \text { and } \int_{M} f d v_{g}=0, \int_{M} f f_{j} d v_{g}=0,\right. \\
\text { where } \left.L_{T} f_{j}=-\lambda_{j}{ }^{L_{T}} f_{j}, f_{j} \in C^{\infty}(M), j=1, \ldots, i-1\right\}
\end{gathered}
$$

is the $i$ th nonzero eigenvalue $(i=2, \ldots, n)$.

Let $o \in \bar{M}^{m}$ and let $\exp _{o}$ be the exponential map at this point, let $\left\{x_{A}\right\}_{1 \leq A \leq m}$ be the normal coordinates centered in $o$, with respect to some orthonormal basis in $T_{o}\left(\bar{M}^{m}\right)$, and $s(\cdot)=d(\cdot, o)$ be the distance function from $o$ in $\bar{M}^{m}$; if $c>0$ we assume that $\phi(M)$ is contained in a convex ball of radius less than or equal to $\pi / 2 \sqrt{c}$. Let $S_{c}(s), \theta_{c}(s)$ be functions defined by

$$
S_{c}(s)= \begin{cases}\frac{1}{\sqrt{c}} \sin \sqrt{c} s, & c>0 \\ s, & c=0 \\ \frac{1}{\sqrt{-c}} \sinh \sqrt{-c} s, & c<0,\end{cases}
$$

and $\theta_{c}(s)=\left(d / d_{s}\right) S_{c}(s)$. Obviously

$$
\theta_{c}^{2}(s)+c S_{c}^{2}(s)=1 \quad \text { and } \quad \theta^{\prime}(s)=-c S_{c}(s) .
$$

Define the generalized position vector field $X$ of $M^{n}$ in $\bar{M}^{m}$, with respect to $o$, by $X=S_{c}(s) \bar{\nabla} s$, it is easy to see that its coordinates in the normal local frame are $\left\{\left(S_{c}(s) / s\right) x_{A}\right\}_{1 \leq A \leq m}$.

REMARK 2.1. In the case $c=0, X=S_{c}(s) \bar{\nabla} s$ is just the position vector field in $m$ Euclidean space $\mathbb{E}^{m}$.

Lemma 2.2. For $x \in \bar{M}^{m}$, and in the case $c>0, x \in B(o, \pi / 2 \sqrt{c})$. Then for any $u \in T_{x}\left(\bar{M}^{m}\right)$, we have

$$
\sum_{A=1}^{m}\left[\bar{g}_{x}\left(\bar{\nabla} x_{A}, u\right)\right]^{2} \leq \frac{s^{2}}{S_{c}^{2}} \bar{g}_{x}(u, u)+\left(1-\frac{s^{2}}{S_{c}^{2}}\right)\left[\bar{g}_{x}(u, \bar{\nabla} s)\right]^{2}
$$

and equality holds when $\left(\bar{M}^{m}, \bar{g}\right)$ is a constant curvature space with sectional curvature $c$. 
Proof. Let $\exp _{o} \tilde{x}=x, \tilde{x} \in T_{o}\left(\bar{M}^{m}\right)$, then the $\operatorname{map}\left(d \exp _{o}\right) \tilde{x}: T_{o}\left(\bar{M}^{m}\right) \rightarrow T_{x}\left(\bar{M}^{m}\right)$ is a linear isomorphism. Let $\gamma:[0, s] \rightarrow \bar{M}^{m}$ be a normalized geodesic with $\gamma(0)=o, \quad \gamma(s)=x, \quad \gamma^{\prime}(0)=\tilde{x} /|\tilde{x}|$, where $|\tilde{x}|=s=\left[\sum_{A=1}^{m} x_{A}^{2}\right]^{1 / 2}$, let $v=u-$ $\bar{g}_{x}(u, \bar{\nabla} s) \bar{\nabla} s \in T_{x}\left(\bar{M}^{m}\right)$, then $v$ is orthogonal to $\bar{\nabla} s$.

We use the notation $\widetilde{v}=\left[\left(d \exp _{o}\right) \widetilde{x}\right]^{-1} v \in T_{o}\left(\bar{M}^{m}\right)$; by the standard Jacobi field estimate [5,9], we have $|\widetilde{v}| \leq s|v| / S_{c}(s)$, and equality holds when $\left(\bar{M}^{m}, \bar{g}\right)$ is a constant curvature space.

Using $\bar{g}\left(\bar{\nabla} x_{A}, v\right)=v\left(x_{A}\right)=\left[\left(d \exp _{o}\right)_{\tilde{x}}^{-1} v\right]\left(x_{A}\right)$, we obtain

$$
\sum_{A=1}^{m} \bar{g}\left(\bar{\nabla} x_{A}, v\right)^{2}=\left|\left(d \exp _{o}\right)_{\widetilde{x}}^{-1} v\right|^{2}=|\widetilde{v}|^{2} \leq \frac{s^{2}}{S_{c}^{2}(s)}|v|^{2} .
$$

On the other hand,

$$
\begin{aligned}
\sum_{A=1}^{m} \bar{g}_{x}\left(\bar{\nabla} x_{A}, u\right) \bar{g}_{x}\left(\bar{\nabla} x_{A}, \bar{\nabla} s\right) & =\bar{g}_{x}\left(\left(d \exp _{o}\right)_{\tilde{x}}^{-1} u,\left(d \exp _{o}\right)_{\tilde{x}}^{-1}(\bar{\nabla} s)\right) \\
& =\bar{g}_{x}\left(\left(d \exp _{o}\right)_{\tilde{x}}^{-1}\left(\bar{g}_{x}(u, \bar{\nabla} s) \bar{\nabla} s\right),\left(d \exp _{o}\right)_{\widetilde{x}}^{-1}(\bar{\nabla} s)\right) \\
& =\bar{g}_{x}(u, \bar{\nabla} s) \bar{g}_{x}\left(\left(d \exp _{o}\right)_{\tilde{x}}^{-1}(\bar{\nabla} s),\left(d \exp _{o}\right)_{\tilde{x}}^{-1}(\bar{\nabla} s)\right) \\
& =\bar{g}_{x}(u, \bar{\nabla} s)
\end{aligned}
$$

so

$$
\sum_{A=1}^{m}\left[\bar{g}_{x}\left(\bar{\nabla} x_{A}, u-\bar{g}_{x}(u, \bar{\nabla} s) \bar{\nabla} s\right)\right]^{2}=\sum_{A=1}^{m} \bar{g}_{x}\left(\bar{\nabla} x_{A}, u\right)^{2}-\bar{g}_{x}(u, \bar{\nabla} s)^{2} .
$$

By (2.3) and the above formula, we have

$$
\begin{aligned}
\sum_{A=1}^{m}\left[\bar{g}_{x}\left(\bar{\nabla} x_{A}, u\right)\right]^{2} & \leq \frac{s^{2}}{S_{c}^{2}(s)}\left|u-\bar{g}_{x}(u, \bar{\nabla} s) \bar{\nabla} s\right|^{2}+\left[\bar{g}_{x}(u, \bar{\nabla} s)\right]^{2} \\
& =\frac{s^{2}}{S_{c}^{2}(s)}\left(|u|^{2}-\left[\bar{g}_{x}(u, \bar{\nabla} s)\right]^{2}\right)+\left[\bar{g}_{x}(u, \bar{\nabla} s)\right]^{2} \\
& =\frac{s^{2}}{S_{c}^{2}(s)}|u|^{2}+\left(1-\frac{s^{2}}{S_{c}^{2}(s)}\right)\left[\bar{g}_{x}(u, \bar{\nabla} s)\right]^{2} .
\end{aligned}
$$

We can easily see that all of the inequalities above are in fact equalities if $\left(\bar{M}^{m}, \bar{g}\right)$ is of constant sectional curvature $c$.

Let $X^{\top}, X^{\perp}$ be the tangential and the normal projection of $X$ respectively on the tangent bundle and the normal bundle of $M^{n}$. Grosjean [5] proved an important inequality

$$
\operatorname{div}_{M}\left(T X^{\top}\right) \geq(\operatorname{tr}(T)) \theta_{c}(s)+\bar{g}\left(X, H_{T}\right)
$$

where the equality holds if $T$ is the identity and $\left(\bar{M}^{m}, \bar{g}\right)$ has a constant sectional curvature equal to $c$. 
Now we improve and simplify the proof process of (2.4), and obtain the fact that the equality holds if $\left(\bar{M}^{m}, \bar{g}\right)$ has a constant sectional curvature equal to $c$, that is, the condition that $T$ is the identity can be omitted.

LEMMA 2.3. For all symmetric divergence-free positive-definite (1.1)-tensors $T$ on $M^{n}$, we have the inequality (2.4), and the equality holds if $\left(\bar{M}^{m}, \bar{g}\right)$ has a constant sectional curvature equal to $c$.

PROOF. For $x \in \bar{M}$, let $\left\{e_{i}\right\}_{1 \leq i \leq n}$ be an arbitrary local orthonormal frame at $x$, by using the standard Jacobi field estimates (see [9, Lemma 2.9, p. 153]), we have for all vectors $v$ orthogonal to $\bar{\nabla} s$ at $x$, the inequality

$$
\bar{g}_{x}\left(\bar{\nabla}_{v} \bar{\nabla} s, v\right) \geq \frac{\theta_{c}}{S_{c}}|v|_{x}^{2}
$$

and equality holds if $\bar{M}$ has a constant sectional curvature equal to $c$.

Similar to the method applied in the proof of Lemma 2.2, for any $u \in T_{x}(\bar{M})$, let $v=u-\bar{g}_{x}(u, \bar{\nabla} s) \bar{\nabla} s$, by direct calculation we can obtain

$$
\bar{g}_{x}\left(\bar{\nabla}_{u} \bar{\nabla} s, u\right) \geq \frac{\theta_{c}}{S_{c}}\left\{|u|_{x}^{2}-\left[\bar{g}_{x}(u, \bar{\nabla} s)\right]^{2}\right\} .
$$

So it follows that

$$
\begin{aligned}
\sum_{i=1}^{n} \bar{g}_{x}\left(\bar{\nabla}{ }_{\sqrt{T} e_{i}} \bar{\nabla} s, \sqrt{T} e_{i}\right) & \geq \frac{\theta_{c}}{S_{c}} \sum_{i=1}^{n}\left\{\bar{g}_{x}\left(\sqrt{T} e_{i}, \sqrt{T} e_{i}\right)-\left[\bar{g}_{x}\left(\sqrt{T} e_{i}, \bar{\nabla} s\right)\right]^{2}\right\} \\
& =\frac{\theta_{c}}{S_{c}} \sum_{i=1}^{n}\left\{\bar{g}_{x}\left(T e_{i}, e_{i}\right)-\left[\bar{g}_{x}\left(\sqrt{T}(\bar{\nabla} s)^{T}, e_{i}\right)\right]^{2}\right\} \\
& =\frac{\theta_{c}}{S_{c}}\left[\operatorname{tr} T-\bar{g}_{x}\left(\sqrt{T}(\bar{\nabla} s)^{T}, \sqrt{T}(\bar{\nabla} s)^{T}\right)\right] \\
& =\frac{\theta_{c}}{S_{c}}\left[\operatorname{tr} T-\bar{g}_{x}\left(T(\bar{\nabla} s)^{T},(\bar{\nabla} s)^{T}\right)\right] .
\end{aligned}
$$

By [5, Equations (14) and (15)],

$$
\begin{aligned}
\operatorname{div}_{M} T X^{\top} & =\bar{g}_{x}\left(X, H_{T}\right)+\theta_{c} \bar{g}_{x}\left(T(\bar{\nabla} s)^{T},(\bar{\nabla} s)^{T}\right)+S_{c} \sum_{i=1}^{n} \bar{g}_{x}\left(\bar{\nabla}_{\sqrt{T} e_{i}} \bar{\nabla} s, \sqrt{T} e_{i}\right) \\
& \geq \bar{g}_{x}\left(X, H_{T}\right)+\theta_{c}(\operatorname{tr} T)
\end{aligned}
$$

and the equality holds if $\left(\bar{M}^{m}, \bar{g}\right)$ has a constant sectional curvature equal to $c$.

Corollary 2.4. Let $f(s)$ be a positive and $C^{k}(k \geq 1)$ function, where $s(\cdot)=d(\cdot, o)$ is the distance function in $\bar{M}^{m}$, then

$$
\int_{M} \frac{f^{\prime}(s)}{S_{c}(s)} g_{x}\left(T X^{\top}, X^{\top}\right) d v \leq \int_{M} f(s)\left|H_{T}\right|\left|X^{\perp}\right| d v-\int_{M}(\operatorname{tr} T) \theta_{c} f(s) d v
$$

and equality holds if $\left(\bar{M}^{m}, \bar{g}\right)$ is a constant curvature space. 
PROOF. Using

$\operatorname{div}_{M}\left(f(s) T X^{\top}\right)=f(s) \operatorname{div}_{M} T X^{\top}+g_{x}\left(T X^{\top}, \nabla^{M} f(s)\right), \quad \nabla^{M} f(s)=\frac{f^{\prime}(s)}{S_{c}(s)} X^{\top}$,

the proof follows easily from the inequality (2.4), the divergence theorem, and the compactness of $M^{n}$.

\section{Proofs of the theorems}

Proof of Theorem 1.1. For any $p \in \phi\left(M^{n}\right) \subset \bar{M}^{m}$, let $\left\{e_{1}, e_{2}, \ldots, e_{m}\right\}$ be an orthonormal basis of $T_{p}\left(\bar{M}^{m}\right)$, using the compactness of $M^{n}$ and the assumption that $\phi\left(M^{n}\right)$ is contained in a convex ball $B$ of radius $\pi / 4 \sqrt{c}$, by a standard argument $[4,5]$ we can parallel translate the frame $\left\{e_{1}, e_{2}, \ldots, e_{m}\right\}$ along every geodesic emanating from $p$ and thereby obtain a differentiable orthonormal frame field $\left\{E_{1}, E_{2}, \ldots, E_{m}\right\}$ in a neighborhood of $B$. We define a vector field near $B$ as

$$
Y_{q} \triangleq \int_{M} \frac{S_{c}(s(q, p))}{s(q, p)} \exp _{q}^{-1}(p) d v_{p} \in T_{q}\left(\bar{M}^{m}\right)
$$

which points towards the interior of $B$ at the boundary $\partial B$. Thus, by the Brouwer fixed-point theorem and the continuity of $\left.Y_{q}\right|_{B}$, there exists a point $o \in B$, such that $Y_{o}=0$; that is

$$
\int_{M} \frac{S_{c}(s)}{s} x_{A} d v_{p}=0
$$

where $\left\{x_{A}\right\}$ is the normal coordinates with respect to $o$.

Since $M^{n}$ is contained in a convex ball $B$ of radius $\pi / 4 \sqrt{c}$, this means that $M^{n}$ lies in a convex ball $\widetilde{B}$ of radius $\pi / 2 \sqrt{c}$ around $o$, with $c>0$.

By

$$
s=|X|=\left[\sum_{A=1}^{m}\left(x_{A}\right)^{2}\right]^{1 / 2}, \quad s \bar{\nabla} s=\sum_{A=1}^{m} x_{A} \bar{\nabla} x_{A}
$$

and $\nabla^{M} S_{c}=\left(\bar{\nabla} S_{c}\right)^{T}=\theta_{c} \nabla^{M} s$, we have

$$
\nabla^{M}\left(\frac{S_{c}}{s} x_{A}\right)=\frac{x_{A}}{s}\left(\theta_{c}-\frac{S_{c}}{s}\right) \nabla^{M} s+\frac{S_{c}}{s} \nabla^{M} x_{A} .
$$

On the other hand,

$$
X^{\top}=\left(S_{c}(s) \bar{\nabla} s\right)^{T}=S_{c}(s) \nabla^{M} s
$$


Using Lemma 2.2, (3.1) and (3.3) we obtain

$$
\begin{aligned}
\lambda_{1}^{T} \int_{M}|X|^{2} d v_{g}= & \lambda_{1}^{T} \int_{M} \sum_{A=1}^{m}\left(\frac{S_{c}}{s} x_{A}\right)^{2} d v_{g} \\
\leq & \sum_{A=1}^{m} \int_{M} g_{x}\left(T \nabla^{M}\left(\frac{S_{c}}{s} x_{A}\right), \nabla^{M}\left(\frac{S_{c}}{s} x_{A}\right)\right) d v_{g} \\
= & \sum_{A=1}^{m} \int_{M} \frac{x_{A}^{2}}{s^{2}}\left(\theta_{c}-\frac{S_{c}}{s}\right)^{2} g_{x}\left(T \nabla^{M} s, \nabla^{M} s\right) d v_{g} \\
& \quad+2 \sum_{A=1}^{m} \int_{M} \frac{x_{A}}{s^{2}} S_{c}\left(\theta_{c}-\frac{S_{c}}{s}\right) g_{x}\left(T \nabla^{M} s, \nabla^{M} x_{A}\right) d v_{g} \\
& \quad+\sum_{A=1}^{m} \int_{M} \frac{S_{c}^{2}}{s^{2}} g_{x}\left(T \nabla^{M} x_{A}, \nabla^{M} x_{A}\right) d v_{g} \\
= & \int_{M}\left(\theta_{c}^{2}-\frac{S_{c}^{2}}{s^{2}}\right) g_{x}\left(T \nabla^{M} s, \nabla^{M} s\right) d v_{g} \\
& \quad+\sum_{A=1}^{m} \int_{M} \frac{S_{c}^{2}}{s^{2}} g_{x}\left(T \nabla^{M} x_{A}, \nabla^{M} x_{A}\right) d v_{g} .
\end{aligned}
$$

Since $T$ is a positive symmetric (1.1)-tensor, we can define a natural positive symmetric (1.1)-tensor $\sqrt{T}$ on $M^{n}$, such that $T=\sqrt{T} \sqrt{T}$ (see [5]), we have

$$
\begin{aligned}
\frac{S_{c}^{2}}{s^{2}} \sum_{A=1}^{m} g_{x}\left(T \nabla^{M} x_{A}, \nabla^{M} x_{A}\right) & =\frac{S_{c}^{2}}{s^{2}} \sum_{A=1}^{m} g_{x}\left(\sqrt{T} \nabla^{M} x_{A}, \sqrt{T} \nabla^{M} x_{A}\right) \\
& =\frac{S_{c}^{2}}{s^{2}} \sum_{A=1}^{m} \sum_{i=1}^{n}\left[g_{x}\left(\sqrt{T} \nabla^{M} x_{A}, e_{i}\right)\right]^{2} \\
& =\frac{S_{c}^{2}}{s^{2}} \sum_{A=1}^{m} \sum_{i=1}^{n}\left[\bar{g}_{x}\left(\bar{\nabla} x_{A}, \sqrt{T} e_{i}\right)\right]^{2} \\
\leq & \sum_{i=1}^{n} \bar{g}_{x}\left(\sqrt{T} e_{i}, \sqrt{T} e_{i}\right) \\
& +\sum_{i=1}^{n}\left(\frac{S_{c}^{2}}{s^{2}}-1\right)\left[\bar{g}_{x}\left(\sqrt{T} e_{i}, \bar{\nabla} s\right)\right]^{2} \\
& \sum_{i=1}^{n} g_{x}\left(T e_{i}, e_{i}\right)+\left(\frac{S_{c}^{2}}{s^{2}}-1\right) \sum_{i=1}^{n}\left[g_{x}\left(\sqrt{T} \nabla^{M} s, e_{i}\right)\right]^{2} \\
& =\operatorname{tr} T+\left(\frac{S_{c}^{2}}{s^{2}}-1\right) g_{x}\left(T \nabla^{M} s, \nabla^{M} s\right) .
\end{aligned}
$$


Furthermore, from (3.5), we have

$$
\lambda_{1}^{T} \int_{M}|X|^{2} d v_{g} \leq \int_{M} \operatorname{tr} T d v_{g}-c \int_{M} g_{x}\left(T X^{\top}, X^{\top}\right) d v_{g} .
$$

Let $\bar{\theta}_{c}=1 / V \int_{M} \theta_{c} d v_{g}$, then we obtain

$$
\int_{M}\left(\theta_{c}-\bar{\theta}_{c}\right) d v_{g}=0
$$

Using $\nabla^{M} \theta_{c}=-c X^{\top}$, and the Rayleigh quotient with the test function $\theta_{c}-\bar{\theta}_{c}$, we obtain

$$
\begin{aligned}
& \lambda_{1}^{T} \int_{M}\left(\theta_{c}-\bar{\theta}_{c}\right)^{2} d v_{g} \\
& \quad \leq \int_{M} g_{x}\left(T \nabla^{M}\left(\theta_{c}-\bar{\theta}_{c}\right), \nabla^{M}\left(\theta_{c}-\bar{\theta}_{c}\right)\right) d v_{g}=c^{2} \int_{M} g_{x}\left(T X^{\top}, X^{\top}\right) d v_{g} .
\end{aligned}
$$

Thus,

$$
\lambda_{1}^{T} \int_{M} \theta_{c}^{2} d v_{g} \leq c^{2} \int_{M} g_{x}\left(T X^{\top}, X^{\top}\right) d v_{g}+\lambda_{1}^{T} \frac{1}{V}\left(\int_{M} \theta_{c} d v_{g}\right)^{2} .
$$

By (3.6) and $\theta_{c}^{2}+c S_{c}^{2}=1$, we have

$$
\lambda_{1}^{T} V \leq c \int_{M} \operatorname{tr} T d v_{g}+\frac{\lambda_{1}^{T}}{V}\left(\int_{M} \theta_{c} d v_{g}\right)^{2} .
$$

Let $f(s)=$ constant $>0$ in Corollary 2.4, then we obtain

$$
\int_{M} \theta_{c} \operatorname{tr} T d v_{g} \leq \int_{M}\left|H_{T}\right|\left|X^{\perp}\right| d v_{g} .
$$

From (3.6), for any $S \in \mathcal{A}$, we have

$$
\begin{aligned}
\lambda_{1}^{T} \inf _{M}(\operatorname{tr} S)^{2}\left(\int_{M} \theta_{c} d v_{g}\right)^{2} & \leq \lambda_{1}^{T}\left(\int_{M}\left|H_{S}\right|\left|X^{\perp}\right| d v_{g}\right)^{2} \\
& \leq \lambda_{1}^{T} \int_{M}\left|H_{S}\right|^{2} d v_{g} \int_{M}\left|X^{\perp}\right|^{2} d v_{g} \\
& \leq \int_{M}\left|H_{S}\right|^{2} d v_{g} \int_{M} \operatorname{tr} T d v_{g} .
\end{aligned}
$$

Putting this into (3.8) gives the desired result (1.5), and the equality holds if $\left(\bar{M}^{m}, \bar{g}\right)$ is a constant curvature space of sectional curvature $c$ and $X^{\top}=S_{c}(s) \nabla^{M} s=0$, that is, $\phi(M)$ is contained in a geodesic hypersphere of $\bar{M}^{m}$ centered at $o$. 
Proof OF TheOREM 1.2. Similar to the proof in [5], let $f(s)=\theta_{c}(s)$ in Corollary 2.4 , then we have

$$
c \int_{M} g_{x}\left(T X^{\top}, X^{\top}\right) d v_{g} \geq \int_{M} \theta_{c}^{2}(\operatorname{tr} T) d v_{g}-\int_{M}\left|H_{T}\right|\left|X^{\perp}\right| \theta_{c} d v_{g} .
$$

By (3.6), for any $S \in \mathcal{A}$, we immediately obtain

$$
\begin{aligned}
\lambda_{1}^{T} \int_{M} S_{c}^{2} d v_{g} & \leq \int_{M} \operatorname{tr} T d v_{g}-\int_{M}\left[\theta_{c}^{2}(\operatorname{tr} T)-\left|H_{T}\right| \theta_{c}\left|X^{\perp}\right|\right] d v_{g} \\
& =c \int_{M} S_{c}^{2}(\operatorname{tr} T) d v_{g}+\int_{M} \theta_{c}\left|H_{T}\right|\left|X^{\perp}\right| d v_{g} \\
& \leq c \int_{M} S_{c}^{2}(\operatorname{tr} T) d v_{g}+\sup _{M}\left(\frac{\left|H_{T}\right|}{\operatorname{tr} S}\right) \int_{M} \theta_{c} \operatorname{tr} S\left|X^{\perp}\right| d v_{g}
\end{aligned}
$$

Taking $f(s)=S_{c}(s)$ in Corollary 2.4

$$
\int_{M}(\operatorname{tr} T) \theta_{c} S_{c} d v_{g} \leq \int_{M}\left|H_{T}\right| S_{c}\left|X^{\perp}\right| d v_{g}-\int_{M} \frac{\theta_{c}(s)}{S_{c}(s)} g_{x}\left(T X^{\top}, X^{\top}\right) d v_{g} .
$$

By the positive definiteness of $T$ and (3.10),

$$
\lambda_{1}^{T} \int_{M} S_{c}^{2} d v_{g} \leq c \int_{M} S_{c}^{2}(\operatorname{tr} T) d v_{g}+\sup _{M}\left(\frac{\left|H_{T}\right|}{\operatorname{tr} S}\right) \int_{M}\left|H_{S}\right| S_{c}^{2} d v_{g},
$$

that is,

$$
\lambda_{1}^{T} \leq \sup _{M}\left[c \operatorname{tr} T+\sup _{M}\left(\frac{\left|H_{T}\right|}{\operatorname{tr} S}\right)\left|H_{S}\right|\right], \quad \text { for all } S \in \mathcal{A} .
$$

So the equality holds if $\left(\bar{M}^{m}, \bar{g}\right)$ is a constant curvature space of sectional curvature $c$ and $X^{\top}=S_{c}(s) \nabla^{M} s=0$; that is, $\phi(M)$ is contained in a geodesic hypersphere of $\bar{M}^{m}$ centered at $o$.

\section{Application to the operator $L_{r}$}

Let $M^{n}$ be a connected, orientable and compact manifold without boundary isometrically immersed in space form $\mathbb{R}^{n+1}(c)$, we now introduce the $(1,1)$-type Newton tensor $T_{r}^{[1],[2]}$ by

$$
\begin{aligned}
T_{0} & =I, \\
T_{1} & =\sigma_{1} I-A, \\
& \vdots \\
T_{r} & =\sigma_{r} I-\sigma_{r-1} A+\cdots+(-1)^{k} \sigma_{r-k} A^{k}+\cdots+(-1)^{r} A^{r},
\end{aligned}
$$


or inductively by $T_{r}=\sigma_{r} I-A I_{r-1}(r=1, \ldots, n)$, where $A$ is the second fundamental tensor of the isometric immersion. Associated with each $T_{r}$, we have on $M^{n}$ a second-order self-adjoint differential operator $L_{r}$ defined by

$$
L_{r} f=\operatorname{div}\left(T_{r} \nabla^{M} f\right),
$$

where $\operatorname{div}_{M}$ and $\nabla^{M}$ are the divergence and the gradient of the metric $g$. On the other hand, by the Codazzi formula, as proved by Rosenberg [7]

$$
\operatorname{div}_{M} T_{r}=\operatorname{trace}\left(\nabla^{M} T_{r}\right)=\sum_{i=1}^{n}\left(\nabla_{e_{i}}^{M} T_{r}\left(e_{i}\right)\right)=0 .
$$

So the $L_{r}$ operator can also be given by

$$
L_{r} f=\operatorname{trace}\left(T_{r} \operatorname{Hess}(f)\right)
$$

for each $r=0,1, \ldots, n$.

In the case $r=0, L_{0}=\Delta$ is naturally elliptic operator, but $L_{r}(r \geq 1)$ is not usually elliptic, the following Lemma 4.1 proves that $L_{r}$ is elliptic under certain hypotheses.

LEMMA 4.1 (Barbosa and Colares [3]). Let $M^{n}$ be a connected, orientable and compact manifold without boundary isometrically immersed by $\phi$ into space form $\mathbb{R}^{n+1}(c)$, in the case $c>0$ we assume that $\phi(M)$ is contained in an open hemisphere of the Euclidean sphere $\mathbb{R}^{n+1}(c)$. If $H_{r+1}>0$, then for each $j(1 \leq j \leq r)$, we have $j$-mean curvature $H_{j}>0$ and $L_{j}$ is elliptic.

Therefore, when $H_{r+1}>0, T_{r} \in \mathcal{A}$, using the relations $\operatorname{tr} T=\operatorname{tr} T_{r}=c_{r} H_{r}$ and

$$
\left|H_{T}\right|=\sum_{1 \leq i \leq n} B\left(T e_{i}, e_{i}\right)=\sum_{1 \leq i \leq n} g\left(A T\left(e_{i}\right), e_{i}\right)=\operatorname{tr}(A T)=c_{r} H_{r+1}
$$

(see [3]). We immediately have the following results by applying Theorems 1.1 and 1.2 to $T_{r}$.

COROllary 4.2. Let $M^{n}$ be a connected, orientable and closed manifold isometrically immersed by $\phi$ into space form $\mathbb{R}^{n+1}(c)(c>0)$, and $\phi(M)$ is contained in a convex ball of radius less than or equal to $\pi / 4 \sqrt{c}$, if there exists a non-negative integer $r(r=0,1, \ldots, n-1)$, such that $H_{r+1}>0$, then

$$
\lambda_{1}^{L_{r}} \leq \frac{c_{r} \int_{M} H_{r} d v_{g}}{V}\left[c+\frac{1}{V} \frac{1}{\inf _{M} H_{s}^{2}} \int_{M} H_{s+1}^{2} d v_{g}\right], \quad \text { for all } s=0,1,2, \ldots, r
$$

equality holds if and only if $\phi(M)$ is a geodesic hypersphere in $\mathbb{R}^{n+1}(c)$.

Corollary 4.3. Let $M^{n}$ be a connected, orientable and closed manifold isometrically immersed by $\phi$ into space form $\mathbb{R}^{n+1}(c)$; in the case $c>0$ we assume that $\phi(M)$ is contained in a convex ball of radius less than or equal to $\pi / 4 \sqrt{c}$, 
if there exists a nonnegative integer $r(r=0,1, \ldots, n-1)$, such that $H_{r+1}>0$, then we have

$$
\lambda_{1}^{L_{r}} \leq c_{r} \sup _{M}\left[c H_{r}+\sup _{M}\left(\frac{H_{r+1}}{H_{s}}\right) H_{s+1}\right] \text { for all } s=0,1,2, \ldots, r
$$

equality holds if and only if $\phi(M)$ is a geodesic hypersphere in $\mathbb{R}^{n+1}(c)$.

REMARK 4.4. When $\mathbb{R}^{n+1}(c)=\mathbb{S}^{n+1}(c)(c>0)$ or $\mathbb{H}^{n+1}(c)(c<0)$, we improved and obtained the $H_{r}-H_{s}$-type upper bounds of $\lambda_{1}^{L_{r}}$ (see [2]) and the corresponding result in $[1,8]$.

\section{References}

[1] H. Alencar, M. do Carmo and F. Marques, 'Upper bounds for the first eigenvalue of the operator $L_{r}$ and some applications', Illinois J Math. 45 (2001), 851-863.

[2] L. J. Alias and J. M. Malacarne, 'On the first eigenvalue of the linearized operator of the higher order mean curvature for closed hypersurfaces in space forms', Illinois J. Math. 48 (2004), 219240.

[3] J. L. Barbosa and A. G. Colares, 'Stablity of hypersurfaces of constant $r$-mean curvature', Ann. Global Anal. Geom. 15 (1997), 277-297.

[4] I. Chavel, 'On A. Hurwitz' method in isoperimetric inequalities', Proc. Amer. Math. Soc. 71 (1978), 275-279.

[5] J. F. Grosjean, 'Extrinsic upper bounds for the first eigenvalue of elliptic operators', Hokkaido Math. J. 33(2) (2004), 341-355.

[6] R. C. Reilly, 'On the first eigenvalue of the Laplacian for compact submanifolds of Euclidean space', Comment. Math. Helv. 52 (1977), 525-553.

[7] H. Rosenberg, 'Hypersurfaces of constant curvature in space forms', Bull. Sci. Math. 117 (1993), 211-239.

[8] W. Rushan, 'Sharp upper bounds for $\lambda_{1}^{L_{r}}$ of immersed hypersurfaces and their $r$-stability in space forms', Acta. Math. Sinica. (Engl. Ser.) 24(5) (2008), 749-760.

[9] T. Sakai, Riemannian Geometry, Translations of Mathematical Monographs, 149 (American Mathematical Society, Providence, RI, 1996).

RUSHAN WANG, College of Mathematics and Computer Science, Anhui Normal University, Wuhu 241000, PR China

e-mail: wangrs@mail.ahnu.edu.cn 